\title{
Wireless Transfer of Solar Power for Charging Mobile Devices in a Vehicle
}

\author{
M. Bhagat and S. Nalbalwar \\ Dept. of E \& Tc, Dr. B. A. Tech. University, Lonere - 402103, MH, India \\ \{milindpb@gmail.com; nalbalwar_sanjayan@yahoo.com \}
}

\begin{abstract}
This manuscript proposes the low cost, efficient yet effective system for transfer of solar power from photo-voltaic cell to mobile charger using wireless power transfer techniques. The proposed system consists of off-the-shelf electronics components such as Arduino, power MOSFETs, rectifier diodes, etc. We have given the detailed steps for the component selection and thereby design of complete system. We hope that the experimental results of the proposed system aids to efficient transfer of solar power and thus, making mobile devices truly portable.
\end{abstract}

Keywords: Wireless power transfer, strongly coupled magnetic field, solar power, Magnetic Resonance, Mobile charger.

\section{Introduction}

We live in the era where mobile devices like cell phones, tablets, PDAs, laptops etc. became the fourth basic need after food, shelter and clothing's. These devices have made our life more comfortable, efficient and portable. However, unlike three basic needs, forth need is a power hungry. They need to be charged on regular basis. During this period, the meaning of portability is lost completely since it has to be connected to the wall outlet. Moreover, when we are traveling in a vehicle, for example a car, we need to generate the electricity for charging mobile devices using the alternator connected to the axle of that vehicle, which increases fuel consumption of the vehicle. If the power is generated using photo-voltaic (PV) cells on the rooftop, and transferred without wires to the devices, it will keep electrical system independent of the other systems of the vehicle and at the same time reduce the burden of Gasoline consumption.

Advancement in Photo-voltaic cells technology enables a low cost and efficient solar panel that can be mounted on rooftop of houses or vehicles. On the other hand, recent activity of wireless power transfer using strongly coupled magnetic field gives efficient mid-range wireless transfer of electrical power up to 2 to 10 meters [1]. The idea of this manuscript is to propose a system in which solar power is generated at the top of a vehicle (in this case a car) and then it is transferred, using magnetically coupled resonance coils, to the mobile devices. The main contribution of our paper is the formulation of whole system in a cost effective way using off-the-shelf and easily available electrical components.

Section 2 deals with theoretical foundation of the resonance circuit, calculations of the inductance, capacitance and Q-factor, and experimental design. Finally we conclude our paper in section 3.

\section{Theoretical Foundation}

In this section, we provide a chronological review of the development of the wireless power transmission techniques starting from the Nikola Tesla's first wireless power transmission experiment to the strongly coupled magnetic field based mid-range power transfer introduced by Martin Soljacic.

\subsection{Review of Wireless power transmission}

The wireless power transmission systems typically fall into one of two categories; short range, i.e. near-field and long range, i.e. far-field designs. Modern literature on wireless power transmission falls into one of the following three categories: 1) Inductive coupling, 2) Electromagnetic radiation (EMR), and 3) Magnetic resonant coupling.

B. Iyer, S. Nalbalwar and R. Pawade (Eds.)

ICCASP/ICMMD-2016. Advances in Intelligent Systems Research.

Vol. 137, Pp. 673-677.

(C) 2017- The authors. Published by Atlantis Press

This is an open access article under the CC BY-NC license (http://creativecommons.org/licens)es/by-nc/4). 
Inductive coupling When an ac source is connected to a primary of the coil (primary coil), the generated magnetic field induces a voltage across the terminals of the secondary coil of the receiver. A transformer is an example of an inductive coupling. This technique is having high power transfer efficiency for the short-range; it's simple, safe and convenient. Due to these advantages it is used in the applications like, electric toothbrush, the company Splashpower [2] developed magnetic charging pads for cell phones, laptops, and medical implants [3]. The world's first wireless charging standard (Qi) for low-power inductive charging (Less than 5 Watt) has approved by the Wireless Power Consortium in 2010. The inductive coupling can be considered as a mature technology. It is considered as a stepping stone in developments of magnetic resonant coupling for upcoming WPT. Even for the short distance, the output power falls off sharply. It only works better if the nodes are close to each other less than a coil diameter and having perfect alignment for charging direction. Therefore, this technique cannot be used in the wireless sensor networks due to above limitations.

Electromagnetic radiation This technology in wireless power transmission is to development of microwave power transmission [1][4]. Transmitting antenna transmits the electromagnetic radiations to receiving antenna in the form of electromagnetic waves. They are classified into two types as per the energy-emitting direction. One is omni directional and second is unidirectional.

The omni directional transmitter emits electromagnetic waves in ISM band (e.g. 850 - $950 \mathrm{MHz}$ [5] or 902 - 928 $\mathrm{MHz}$ [6] in the United States. Receiver (e.g. RFID tags) harvests the radio power by tuning it to same frequency band. But, the electromagnetic waves decay quickly over distance. The power transfer efficiency is only $\approx 1.5 \%$ when a receiver is $30 \mathrm{~cm}$ away from the RF transmitter [1]. The omni directional radiation is appropriate for ultra low-power sensor nodes (e.g., up to $10 \mathrm{~mW}[4,5]$ ) with very low sensing activities (e.g., temperature, moisture and light) to prevent potential health hazards.

The unidirectional transmitter needs LOS (Line-of-sight) path and it can achieve high power transmission over kilometer range by using laser or microwave. One of the applications, The unmanned plane, SHARP (Stationary High Altitude Relay Platform), 3 flew at an altitude of about $21 \mathrm{~km}$ and in circles of $1 \mathrm{~km}$ in radius so that it could stay in the transmission range of a ground-based transmitter. In microwave-based systems, wireless power is transmitted on microwave frequencies of either 2.45 or $5.8 \mathrm{GHz}$, both in the ISM frequency band. But because of it needs LOS, complicated tracking mechanisms, and the inherent large scale of devices, it is not suitable for wireless sensor networks.

Magnetic resonant coupling Kurs et. al. [1] has developed WPT technology based on magnetic resonant coupling. When two magnetic resonant coils operate at the same frequency, they become strongly coupled by non radiative magnetic resonant induction. Therefore, the energy can be transferred efficiently from a source coil to the receiver coil with negligible energy loss. Magnetic resonant coupling has the higher power transfer efficiency even under omni-direction and does not require LOS. The application, this technology is used to charge multiple devices, mutual coupling among various receiving coils and other objects may cause interference and therefore careful tuning is necessary. It can also be used for charging electric vehicles, implantable devices and the wireless sensor networks.

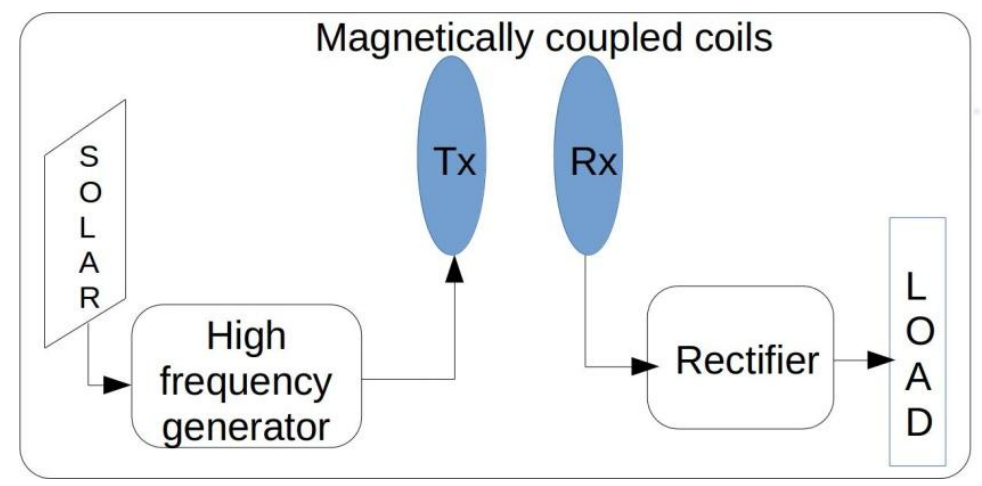

Fig. 1. Experimental prototype for wireless transfer of solar power to mobile charger

\subsection{Resonance circuit theory}

Oersted's experiment demonstrates that, when the current flows through the conductor, the magnetic field of proportional magnitude will be generated around the conductor. The range of the magnetic field (in terms of distance from the coil) depends upon many factors like amount of current passing through the coil, the frequency of the alternating current, etc. 
On the contrary, Faraday's experiment demonstrates that when the conductor coil comes under the influence of varying magnetic field, the emf will be induced in the coil. Modern wireless power transmission technique combined these two experiments to transfer power from one coil to another which are kept at distance from each other. The maximum power efficiency is confirmed when two coils resonate at the same frequency.

In this research work, we are developing a wireless power transmission based system to transfer the solar power generated on the roof top of the vehicle (e.g. a car) to the mobile charger present inside the vehicle. The experimental block diagram of the proposed approach is given in figure 1 . We can generate the DC power using $150 \mathrm{~W}$ solar panel. This solar power will be fed to the ultrafast switching MOSFET. It will generate the high frequency alternating current at $225 \mathrm{KHz}$. The frequency is controlled by simple 8-bit microcontroller using Arduino based platform. The generated high frequency signal is then fed to the parallel L-C circuit, which resonated at the frequency given by the equation (1).

$$
f_{o}=\frac{1}{2 \pi \sqrt{L C}}
$$

When the receiver circuit with same resonance frequency is kept near the transmitter, the emf of same high frequency is generated in the receiving coil, which needs to be converted to DC so that it can be used for charging the mobile devices. Subsequent sections provide guidelines for coil design and component selection.

Calculations of the component values, we need to consider and use various components in the WPT for designing the Tx and Rx coils from which the power is transmitted. For finding the expressions for L, C and Q; number of turns $\mathrm{N}$ with the outermost diameter $\mathrm{D}_{0}$, and inner one is $\mathrm{D}_{\mathrm{i}}$, spacing between the coils is $\mathrm{p}$ and diameter of the coil will be w. The geometric parameters are as shown in the figure given below for the spiral coil. The length of the coil will be 1 , unit is meters.

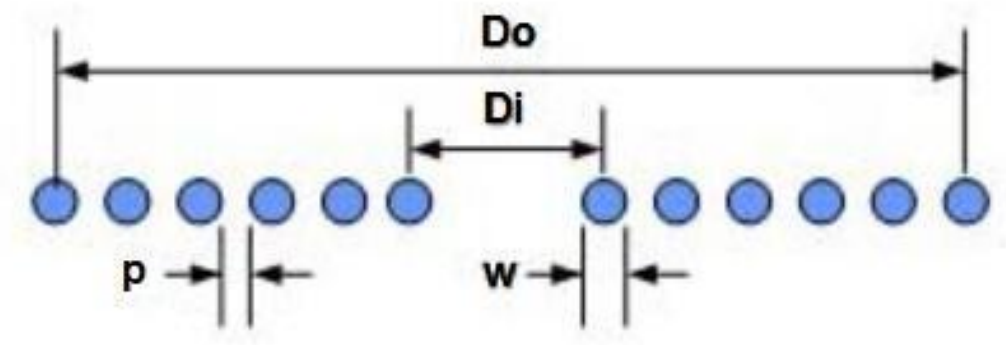

Fig. 2. Spiral Coil Design

$$
\begin{aligned}
& D_{i}=D_{o} 2 N(w+p) \\
& l=\frac{1}{2} N p\left(D_{o}+D_{i}\right)
\end{aligned}
$$

Inductance self inductance for the spiral coils $\mathrm{L}$ is derived from the wheeler's formula for a single-layer helical coil [7],

$$
L(H)=\frac{N^{2}\left(D_{o}-N(w+p)\right)^{2}}{16 D+28 N(w+p)} \times \frac{39.37}{10^{6}}
$$

The inductance since the boundary conditions typically correspond to low inductance (i.e. low Q for a series resonator) and the WPT needs high Q, which lead to inapplicable when designing the coil due to inaccuracy.

Capacitance The self-inductance is in the order of a few micro farads and is small compared to the required tuning capacitor for resonance at $225 \mathrm{KHz}$. Therefore neglecting the parasitic self-capacitance a simple expression for the required capacitance of the coil will be

$$
C(F)=\frac{1}{(2 \pi f)^{2} L}
$$


The relative permittivity of the conductor coil, its diameter of each turn, the pitch and the number of turns will decides the self-capacitance of the spiral coil. As the number of turns increases, the self-capacitance becomes increasingly difficult to calculate accurate due to nonlinear adjacent winding capacitance [8].

Quality factor $\boldsymbol{Q}$ Using $L, C$ and $R, Q$ is defined as

$$
Q=\frac{1}{R} \sqrt{\frac{L}{C}}
$$

Where $R$, is the resistance of the spiral coil. For very high frequency model of AC resistance; Kaiser [9]. This expression can be used to optimize coil design for high $Q$ and ensure resonance at the desired operating frequency.

$$
\begin{gathered}
R_{d c}=\frac{1}{\sigma \pi(w / 2)^{2}}, \quad \delta=\frac{1}{\sqrt{\pi f \sigma \mu_{o}}} \\
R=R_{d c} \frac{\omega}{4 \delta}=\sqrt{\frac{f \pi \mu_{o}}{\sigma}} \frac{N\left(D_{o}-N(w+p)\right)}{\omega}
\end{gathered}
$$

Power loss in a spiral coil consists of losses in radiation and conduction. The WPT coils are relatively small compared to operating wavelength (at $225 \mathrm{KHz}$ ). Thus the conduction loss is the dominate loss mechanism, while radiation loss is negligible. Conduction loss is depends upon proximity effect. The effective resistance of conductor increases due to the current flowing through the smaller cross-sectional areas of the conductor [7].

For the spiral coils, the parameter $p$, the pitch between the turns, that contributes most to fluctuations in total resistance $R$. Which is inversely proportional to $p$, and the effect is nonlinear due to the proximity effect. The accurate expressions for $R$ that accounts for the proximity effect are complex and can be difficult to calculate for tightly wound coils [7]. For loosely coupled wound coils, the proximity effect can be negligible, so $R$ consists of only the DC resistance $R_{d c}$ and the skin depth delta, which can be approximated with a similar expression shown in the above equation. Where $\mu_{o}$ is the permittivity of the free space and delta is the conductivity of the conductor which is equal to $=59.6 \times 106 \mathrm{~s} / \mathrm{m}$ for copper.

\subsection{Experimental Design}

As shown in the figure 3, the solar panel of $(12 \mathrm{~V} / 150 \mathrm{~W})$ has connected as a source. The Arduino is connected for the square wave generation. The simple arduino program is written for the generation of $225 \mathrm{KHz}$ frequency wave. This is fed to the n-channel MOSFET (IRFP250N). The power is fed to the arduino through 7805 voltage regulator and which is tapped from the solar panel by connecting the current limiting resistor in series.

Coil Specifications Coil parameters were optimized using a MATLAB pro-gram for the Tx and Rx coils. Table 1 below the values the parameters calculated to optimize. We have considered the frequency $225 \mathrm{KHz}$ for calculation of inductance. Capacitor used is of the value $0.68 \mu \mathrm{F}$, which gave the value of inductance to be $\mathrm{L}=$ $0.73581 \mu \mathrm{H}$

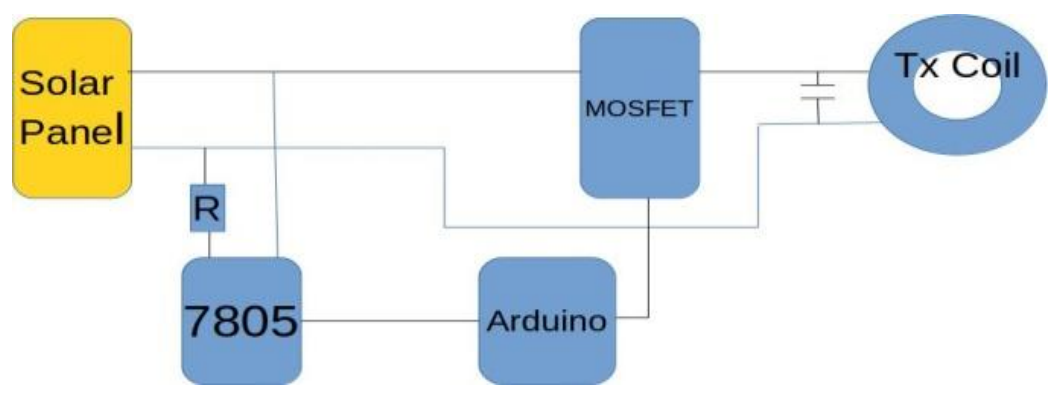

Fig. 3. Experimental setup

\section{$S_{\text {petss }}^{\text {Artants }}$}


Table 1. Coil Specifications

\begin{tabular}{lcc}
\hline & Tx coil & Rx coil \\
\hline Diameter of the wire (w) & $1.016 \mathrm{~mm}$ & $0.315 \mathrm{~mm}$ \\
Spacing between the coils $(\mathrm{p})$ & $20 \mathrm{~mm}$ & $1 \mathrm{~mm}$ \\
Number of Turns $(\mathrm{N})$ & 15 & 33 \\
Outer Diameter (Do) & $350 \mathrm{~mm}$ & $50 \mathrm{~mm}$ \\
Gauge of the copper wire & 19 & 32 \\
Inductance $(0.73581 \mu \mathrm{H})$ & $0.74189 \mu \mathrm{H}$ & $0.74189 \mu \mathrm{H}$ \\
\hline
\end{tabular}

\section{Conclusion}

We proposed a system for wireless power transfer to charge the mobile devices. The system consists of solar panel to generate the DC power, Arduino based microcontroller board to generate high frequency square wave signal, Power MOSFET to Convert DC power from solar panel to high frequency signal generated by Arduino and finally a transmitting coil of appropriate inductance. The power transmitted by the Tx coil is coupled with the Rx coil. At receiving end, this high frequency signal is converted back to DC signal using rectifier, R-C filter and voltage regulator. This regulated DC power is used to charge the mobile devices.

Acknowledgement We thank our colleagues Dr. D. S. Karanjkar, HoD, Instrumentation Engg; Prof. Amit J.Naik, Dept of Electronics and Telecommunication Engg and Rahul G. Waghmare, Research Scholar, IIST, Thiruvananthpuram, who provided insight and expertise that greatly assisted this research work. Their valuable suggestions were of immense help throughout this work.

\section{References}

[1] Andr Kurs, Aristeidis Karalis, Robert Mo att, J. D. Joannopoulos, Peter Fisher, and Marin Soljai. Wireless power transfer via strongly coupled magnetic resonances. Science, 317(5834):83\{86, July 2007.

[2] Scott Mowbray. Best of what's new: Gadgets. Popular Science, 16(12):49, December 2003.

[3] Guoxing Wang, Wentai Liu, M. Sivaprakasam, M. S. Humayun, and J. D. Weiland. Power supply topologies for biphasic stimulation in inductively powered implants. In 2005 IEEE International Symposium on Circuits and Systems, pages 2743\{2746 Vol. 3, 2005.

[4] Aristeidis Karalis, J.D. Joannopoulos, and Marin Soljai. E cient wireless non-radiative mid-range energy transfer. Annals of Physics, 323(1):34\{48, January 2008.

[5] A. P. Sample, D. J. Yeager, P. S. Powledge, A. V. Mamishev, and J. R. Smith. Design of an r d-based battery-free programmable sensing platform. IEEE Transactions on Instrumentation and Measurement, 57(11):2608\{2615, 2008.

[6] S. He, J. Chen, F. Jiang, D. K. Y. Yau, G. Xing, and Y. Sun. Energy provisioning in wireless rechargeable sensor networks. IEEE Transactions on Mobile Computing, 12(10):1931\{1942, 2013.

[7] Frederick W Grover. Inductance Calculations. Dover Publications, 2009.

[8] G. Grandi, M. K. Kazimierczuk, A. Massarini, and U. Reggiani. Stray capacitances of single-layer aircore inductors for high-frequency applications. In Industry Appli-cations Conference, 1996. ThirtyFirst IAS Annual Meeting, IAS '96., Conference Record of the 1996 IEEE, volume 3, pages 1384\{1388 vol.3, 1996.

[9] Kenneth L. Kaiser. Electromagnetic Compatibility Handbook. CRC Press, 2004. 